\title{
Modulation speed improvement in a Fabry-Perot thermo-optical modulator through a driving signal optimization technique
}

\author{
Francesco G. Della Corte \\ Massimo Merenda \\ Mediterranea University of Reggio Calabria \\ Department of Information Science, Mathematics, \\ Electronics and Transportations \\ Via Graziella Feo di Vito \\ Reggio Calabria, 89100 \\ Italy \\ E-mail: massimo.merenda@unirc.it
}

\author{
Giuseppe Cocorullo \\ University of Calabria \\ Department of Electronics, Computer Science \\ and Systems \\ Via Pietro Bucci 42/C \\ Arcavacata di Rende, 87036 \\ Italy
}

\author{
Mario lodice \\ Ivo Rendina \\ Institute for Microelectronics and Microsystems \\ National Council of Research \\ Via Pietro Castellino 111 \\ Naples NA, 80131 \\ Italy \\ Pasqualina M. Sarro \\ Delft University of Technology \\ Delft Institute of Microelectronics and Submicron \\ Technology \\ Feldmanweg 17 \\ Delft, 2628 CT \\ Netherlands
}

\begin{abstract}
A three-power-level method for obtaining efficient thermooptical modulation in an all-silicon waveguide-integrated Fabry-Perot thermo-optic modulator is discussed by means of a thermo-optical analytical model and demonstrated. The thermal system is represented as a two-pole model where, at every time, the temperature in the waveguide core is modified by means of a heater. This temperature is calculated and used in turn for calculating the refractive index. In this way, the impact of the driving signal shape on the device speed performance is assessed. Results clearly indicate that the application of a thermal bias holding the modulator at a higher average temperature with respect to the substrate heat sink allows increasing the modulator speed. An application-specific integrated circuit has been designed and developed in order to test the new modulation logic. The system's electronics is implemented in a $0.8 \mu \mathrm{m}, 5 \mathrm{~V}$, CMOS process. The experimental results of this new three-power-level driver method are reported, showing the shortening of the characteristic transient times. (0) 2009 Society of Photo-Optical Instrumentation Engineers. [DOI: 10.1117/1.3183913]
\end{abstract}

Subject terms: thermo-optical devices; silicon optoelectronics; Fabry-Perot modulators; thermal transients.

Paper 080686RR received Sep. 3, 2008; revised manuscript received May 18, 2009; accepted for publication May 31, 2009; published online Jul. 30, 2009.

\section{Introduction}

Silicon is a favorable candidate to meet low-cost photonic integration requirements. ${ }^{1}$ The thermo-optic effect, explicitly the temperature dependence of the material refractive index, is an usually exploited phenomenon for designing thermo-optic devices (i.e., photonic devices that are operated by inducing temperature changes in the active optical material). Tunable lasers, filters, switches, and modulators are well known examples in literature. Treyz, ${ }^{2}$ for example, proposed a prototype modulator based on a Mach-Zehnder silicon-on-insulator (SOI) structure. This first result showed a low operative speed due to the slowness of the thermal transients, with a bandwidth of a few tens of kilohertz. Later on, Mayer et al. ${ }^{3}$ described a $90-\mathrm{kHz}$ bandwidth Mach-Zehnder modulator based on a SiGe waveguide structure, while Fischer et al. $^{4}$ developed an upgrade of

0091-3286/2009/\$25.00 @ 2009 SPIE
Treyz's device with fastest switching times of $\sim 5 \mu$ s. Better results were achieved with a micromachined all-silicon micromodulator showing a bandwidth beyond $1 \mathrm{MHz} .^{5}$

More recently, a thermo-optically tuned photonic crystal device, based on a SOI waveguide was proposed, reaching $20 \mu$ s switching time. ${ }^{6}$ Finally, several optical switching elements have been demonstrated in polymeric materials, with characteristic times of a few milliseconds. ${ }^{7,8}$

The cooling time is the true speed limit for all thermally activated photonic devices. However, the coupling of opti$\mathrm{cal}$ and thermal simulations of an all-silicon waveguide integrated thermo-optic Fabry-Perot modulator have permitted to understand more in detail the heat exchange mechanism, and the target of reducing the cooling time has been shown to be reachable by using thermal drive schemes alternative to the classical on-off one. In Iodice et al., ${ }^{9}$ for example, the heat transport rate is enhanced by inducing wider temperature gradients between the active volume and 


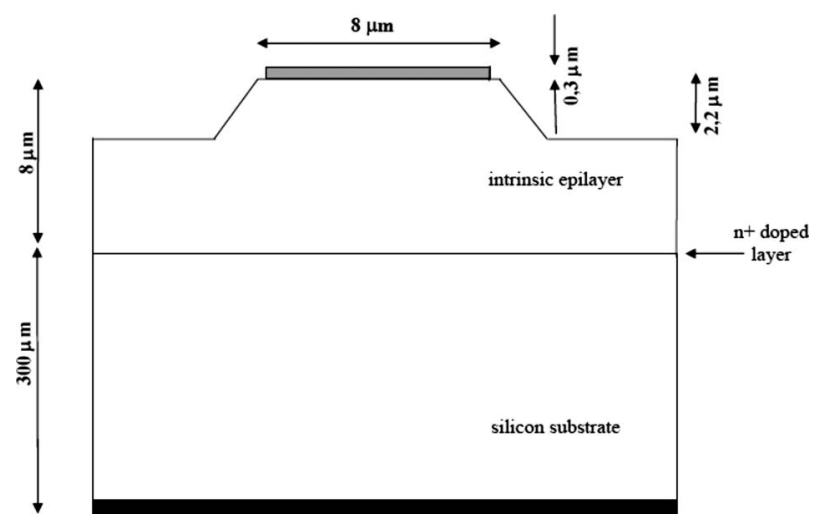

Fig. 1 Cross-sectional view of the waveguide embedding the thermo-optical modulator.

the surrounding medium of the heat sink. In this work, we theoretically analyze the effects of this new kind of driving signal on the speed performances of a waveguide integrated thermo-optic modulator. The analysis is based on a simple lumped element thermal model, from which an approximated transfer function of the system is derived. The thermal model is used for estimating the optical output of the given Fabry-Perot modulator. Experimental data, obtained by means of a custom-designed integrated circuit (IC) developed in a $0.8-\mu \mathrm{m}$ CMOS process, are also presented for comparison.

\section{Thermo-Optical Modulation}

The device considered in this paper for developing the theoretical opto-thermal model is ideally a Fabry-Perot cavity integrated in a rib single-mode silicon waveguide. Vertical trenches in the silicon allow the device to act as an interferometric structure, as demonstrated in a previous work. ${ }^{10}$ In fact, the front and back surfaces delimiting the cavity play the role of two parallel semireflecting mirrors. When a light beam impinges on the front mirror of the cavity, the transmitted intensity $I_{\mathrm{T}}$ can be calculated by the Airy's formula from the incident intensity $I_{0}$

$I_{\mathrm{T}}=I_{0} \frac{1}{1+4\left(F_{\mathrm{R}}^{2} / \pi^{2}\right) \sin ^{2} \phi}$.

The equation is valid for an ideal cavity without optical absorption and for perfectly parallel and smooth mirrors. It is possible to generalize this equation to the more realistic case of lossy waveguides with nonparallel rough mirrors. ${ }^{11}$ In Eq. (1) $\left.F_{\mathrm{R}}=\pi \sqrt{R}\right) /(1-R)$, the reflecting finesse of the cavity, depends on the reflectance $R$ of the air-silicon interface, while $\phi=\left(2 \pi n_{\mathrm{Si}} l\right) / \lambda$ is a phase factor related to the actual refractive index $n_{\mathrm{Si}}$ of silicon, the cavity length $l$, and the wavelength $\lambda$.

When $n_{\mathrm{Si}}$ varies, due for instance, to a temperature variation, a phase shift $\Delta \phi$ is generated that allows a variation of $I_{\mathrm{T}}$ according to Eq. (1). The $\sin 2 \phi$ term produces a periodic pattern of $I_{\mathrm{T}}$ versus the temperature $T$, so that cyclical and equal minima and maxima are present for the transmitted light intensity, namely $I_{\min }$ and $I_{\max }$. Because we desire the modulator output continuously switching be-

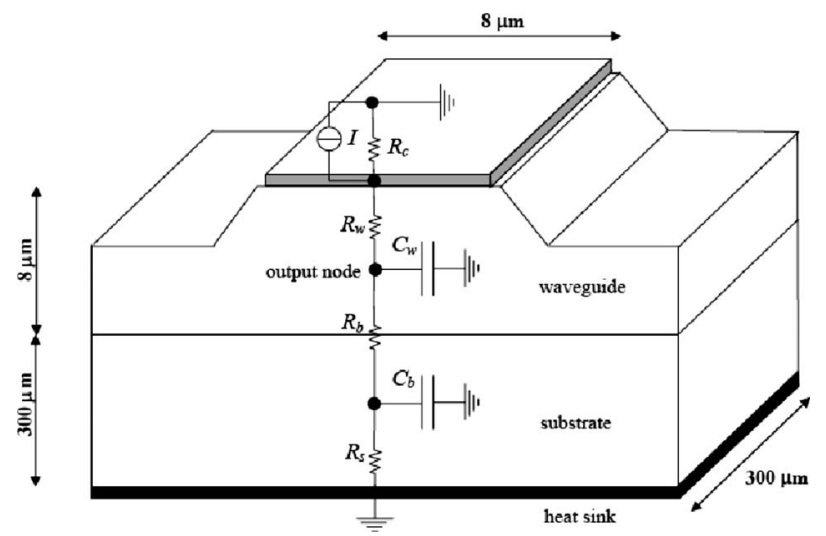

Fig. 2 Illustration of the simple 1-D, lumped element, electrically equivalent thermal model of the modulator. Two thermal capacitances, respectively associated to the waveguide core and to the substrate, are responsible for two distinct time constants in the transients. The figure is not to scale.

tween $I_{\min }$ and $I_{\max }$, this means that the temperature change $\Delta T$ between the respectively associated working points $\left(T_{\max }\right.$ and $\left.T_{\min }\right)$ should produce a phase shift equal to $\pi / 2$. Differentiating $\phi$ with respect to $T$, we obtain

$\frac{\partial \phi}{\partial T}=\frac{2 \pi n_{\mathrm{Si}} l}{\lambda}\left(\frac{1}{n_{\mathrm{Si}}} \frac{\partial n_{\mathrm{Si}}}{\partial T}+\Gamma\right)$,

where $\Gamma=(1 / l)(\partial l / \partial T)=\left(2.6 \times 10^{-6}\right){ }^{\circ} \mathrm{C}^{-1}$ is the material thermal expansion coefficient, ${ }^{12} n_{\mathrm{Si}}=3.47$, and $\partial n_{\mathrm{Si}} / \partial T$ $=\left(1.86 \times 10^{-4}\right){ }^{\circ} \mathrm{C}^{-1}$ obtained in a previous work, ${ }^{13}$ at $\lambda=1.55 \mu \mathrm{m}$, so that from Eq. (2) we have

$\partial \phi / \partial T \cong\left(0.8 \times 10^{-3}\right) l\left[\mathrm{rad}{ }^{\circ} \mathrm{C}^{-1} \mu \mathrm{m}^{-1}\right]$.

Therefore, a $\Delta T \cong 6.7{ }^{\circ} \mathrm{C}$ is required for a $300-\mu \mathrm{m}$-long cavity to obtain $\Delta \phi=\pi / 2$. In this paper, these device length and temperature variations are used in calculations to obtain modulation. The waveguide hosting the modulator is supposed here to be realized on a $300-\mu$ m-thick silicon substrate on which a $8.0-\mu \mathrm{m}$-thick intrinsic epilayer is grown. The vertical light confinement is ensured by a 800-nm-thin $n^{++}$ion-implanted doped layer at the substrateepilayer interface. The lateral confinement is obtained by a 2.2- $\mu \mathrm{m}$-high, 8 - $\mu \mathrm{m}$-wide rib. The geometrical characteristics ensure, in spite of its large cross section, that the waveguide allows single-mode propagation. ${ }^{14}$ The device cross section is sketched in Fig. 1.

A 300-nm-thin doped polysilicon film, deposited onto the upper rib surface, is used as a heat source to produce

Table 1 Material property values.

\begin{tabular}{llll}
\hline \hline$\rho\left(\mathrm{g} / \mathrm{cm}^{3}\right)$ & $c_{\mathrm{p}}\left(\mathrm{J} / \mathrm{g} \cdot{ }^{\circ} \mathrm{C}\right)$ & $k\left(\mathrm{~W} / \mathrm{cm}^{\circ} \mathrm{C}\right)$ & $h\left[{ }^{\circ} \mathrm{C}\left(\mathrm{W} / \mathrm{cm}^{2}\right)^{-1}\right]$ \\
\hline 2.33 & 0.71 & 1.56 & $2 \times 10^{-3}$ \\
\hline
\end{tabular}


Table 2 Approximate values for R's used in the electrically equivalent network (EEN).

\begin{tabular}{lllll}
\hline \hline & $R_{\mathrm{w}}$ & $R_{\mathrm{b}}$ & $R_{\mathrm{s}}$ & $R_{\mathrm{c}}$ \\
\hline$L(\mathrm{~cm})$ & $4 \times 10^{-4}$ & $1.5 \times 10^{-2}$ & $1.5 \times 10^{-2}$ & - \\
$A\left(\mathrm{~cm}^{2}\right)$ & $\left(8 \times 10^{-4}\right) \times\left(3 \times 10^{-2}\right)$ & $\left(8 \times 10^{-4}\right) \times\left(3 \times 10^{-2}\right)$ & $5 \times 10^{-5}$ & $\left(8 \times 10^{-4}\right) \times\left(3 \times 10^{-2}\right)$ \\
Resistance $(\mathrm{K} / \mathrm{W})$ & 11 & 33 & 200 & $2 \times 10^{7}$ \\
\hline \hline
\end{tabular}

the thermo-optic modulation. The heating mechanism source is the power dissipated by the Joule effect. This heating layer is a better choice with regard to a metal cladding that would in fact induce higher attenuation and make the waveguide sensitive to polarization.

\section{Theoretical Approach}

The first step in this work is an analysis of the thermal response characterizing the thermo-optical modulator in order to establish the relationship existing between the driving electrical signal and the output optical signal. From now on, we consider that the modulator is power driven, meaning that at every time we can generate a desired amount of heat in the polysilicon film. This assumption also simplifies the following analysis. We assume that heat flows vertically by conduction from the top heating film to the substrate. Natural convection toward air is also considered, although it will be shown to be negligible. A given temperature at the bottom surface of the modulator chip is assumed, due to the presence of an ideal heat sink. A simple one-dimensional thermal model of the device is considered hereafter. This choice is a trade-off between result accuracy and a fast and direct analysis of the transient response under different driving signals. A one-dimensional lumpedelement electrical equivalent network (EEN) of the modulator thermal model is that shown in Fig. 2.

It is composed of the resistances $R_{\mathrm{w}}, R_{\mathrm{b}}, R_{\mathrm{s}}, R_{\mathrm{c}}$, and the capacitances $C_{\mathrm{w}}$ and $C_{\mathrm{b}}$, where $R_{\mathrm{w}}$ is the thermal resistance between the heating poly-Si film and the waveguide core; $R_{\mathrm{b}}$ is the resistance between the waveguide core and the thermal nucleus of the bulk; $R_{\mathrm{S}}$ is the resistance between the bulk and the external heat sink; and $R_{\mathrm{c}}$ takes into account a heat exchange mechanism through convection between the waveguide and air; $C_{\mathrm{w}}$ and $C_{\mathrm{b}}$ represent the heat storage elements in the waveguide and in the bulk, respectively.

The EEN is driven in our case by a current source, which is the equivalent of a thermal power generator located in the poly-Si film. We consider the voltage amplitude at the node between $R_{\mathrm{w}}$ and $C_{\mathrm{w}}$ as the output of the EEN, corresponding to the temperature in the waveguide thermal center.

Approximate values for $R$ 's and $C$ 's can be calculated as soon as the device dimensions and material properties are known, being, in general, $R=k^{-1} \times L \times A^{-1}$ and $C=\rho \times C_{\mathrm{p}}$ $\times L \times A$, with $k, \rho, C_{\mathrm{p}}$, respectively, the thermal conductivity, the mass density, and the specific heat of silicon (Table 1), and $L$ and $A$ the length and cross-sectional area relevant to the device portion of interest. $R_{\mathrm{c}}$ can be calculated as $(h A)^{-1}$, where $h$ is the silicon-to-air convective heat transfer coefficient. The values of the considered geometrical parameters and of the derived resistances and capacitances are reported in Tables 2 and 3.

For calculating $R_{\mathrm{b}}$, indeed thermal spreading resistance effects were considered. In this case, the heat flow is, in fact, not one-dimensional because it spreads from a planar heat source to an opposing boundary of much larger crosssectional area. The thermal spreading resistance can be calculated using the spreading resistance equation in Ellison ${ }^{15}$

$R=R_{\mathrm{U}}+R_{\mathrm{Sp}}, \frac{\Psi}{k \sqrt{\Delta x \Delta y}}=\frac{\Psi_{\mathrm{U}}}{k \sqrt{\Delta x \Delta y}}+\frac{\Psi_{\mathrm{Sp}}}{k \sqrt{\Delta x \Delta y}}$

where $\Psi$ is the dimensionless total resistance, $\Psi$ $=\Psi_{\mathrm{U}^{+}} \Psi_{\mathrm{Sp} .} \Psi_{\mathrm{U}}$ is the dimensionless 1-D resistance; and $\Psi_{\mathrm{Sp}}$ is the dimensionless spreading resistance

$$
\begin{aligned}
& \Psi_{\mathrm{U}}=\rho \sqrt{\alpha \beta} \tau\left(1+\frac{1}{\mathrm{Biot} \cdot \tau}\right) \\
& \Psi_{\mathrm{Sp}}=\frac{\rho}{\pi^{2}} \sqrt{\frac{\beta}{\alpha}} \sum_{l=1}^{+\infty} \frac{1}{l^{2}} \sin (l \pi \alpha)\left[\frac{1+\left(\frac{\mathrm{Biot} \cdot \tau}{2 l \pi \tau}\right) \tanh (2 l \pi \tau)}{\left(\frac{\mathrm{Biot} \cdot \tau}{2 l \pi \tau}\right)+\tanh (2 l \pi \tau)}\right] \\
& +\frac{1}{\rho \pi^{2}} \sqrt{\frac{\alpha}{\beta}} \sum_{m=1}^{+\infty} \frac{1}{m^{2}} \sin (m \pi \beta \rho)\left[\frac{1+\left(\frac{\mathrm{Biot} \cdot \tau}{m \pi \beta \rho}\right) \tanh (m \pi \beta \rho)}{\left(\frac{\mathrm{Biot} \cdot \tau}{m \pi \beta \rho}\right)+\tanh (m \pi \beta \rho)}\right] \\
& +\frac{4}{\pi^{2} \sqrt{\alpha \beta}} \sum_{l=1}^{+\infty} \sum_{m=1}^{+\infty} \frac{1}{l m} \sin (l \pi \alpha) \sin (m \pi \beta \rho) \\
& \times\left\{\frac{1+\left(\frac{\text { Biot } . \tau}{2 \pi \tau \sqrt{l^{2}+m^{2} \rho^{2}}}\right) \tanh \left(2 \pi \tau \sqrt{l^{2}+m^{2} \rho^{2}}\right)}{2 \pi \tau \sqrt{l^{2}+m^{2} \rho^{2}}\left[\left(\frac{\text { Biot } . \tau}{2 \pi \tau \sqrt{l^{2}+m^{2} \rho^{2}}}\right)+\tanh \left(2 \pi \tau \sqrt{l^{2}+m^{2} \rho^{2}}\right)\right.}\right] .
\end{aligned}
$$

Table 3 Approximate values for $C$ 's used in the EEN.

\begin{tabular}{lll}
\hline \hline & $C_{\mathrm{w}}$ & $C_{\mathrm{b}}$ \\
\hline$L(\mathrm{~cm})$ & $3 \times 10^{-2}$ & $3 \times 10^{-2}$ \\
$A\left(\mathrm{~cm}^{2}\right)$ & $\left(8 \times 10^{-4}\right) \times\left(8 \times 10^{-4}\right)$ & $\left(3 \times 10^{-2}\right) \times\left(3 \times 10^{-2}\right)$ \\
Capacitance $(\mathrm{J} / \mathrm{K})$ & $3.1 \times 10^{-8}$ & $4.5 \times 10^{-5}$ \\
\hline \hline
\end{tabular}




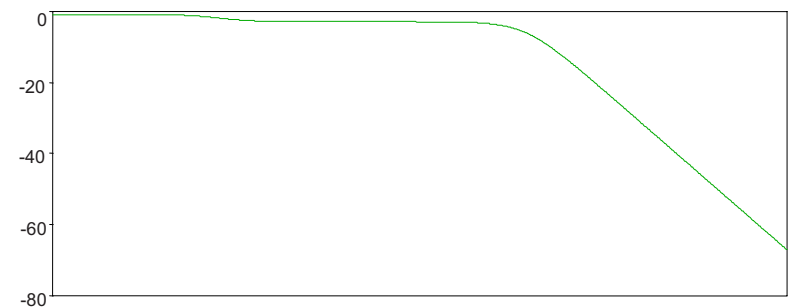

(a)

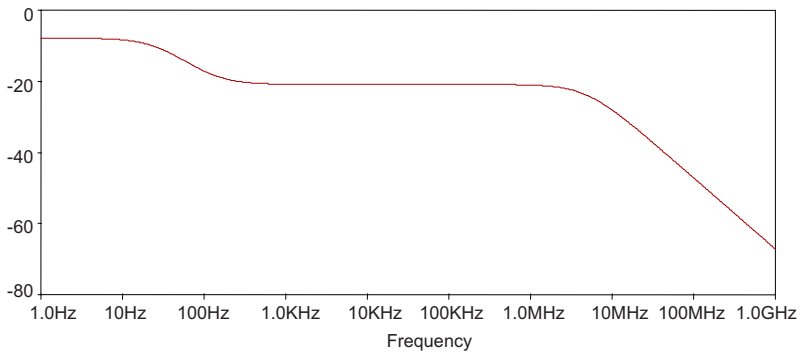

(b)

Fig. 3 (a) Frequency response of the system and (b) frequency response of the system with the prefiltering section. Vertical scale is in decibels $(\mathrm{dB})$.

Assuming $\Delta x=3 \times 10^{-2} \mathrm{~mm}, \quad \Delta y=8 \times 10^{-4} \mathrm{~mm}, \quad t=1.5$ $\times 10^{-2} \mathrm{~mm}$, geometrical parameters of the structure, we get $\Psi_{\mathrm{U}}=0.00633, \Psi_{\mathrm{Sp}}=025$, Biot $=33.3$ and, therefore, $R_{\mathrm{b}}$ $=33 \mathrm{~K} / \mathrm{W}$. This analytical result was furthermore confirmed by a heat transfer analysis performed by numerical simulations. A basic ac analysis, which can be easily performed by a SPICE-like simulation tool, shows that this second-order low-pass network presents two relatively distant poles, one dominated by the large thermal capacitance of the bulk and one bound to the small thermal capacitance of the thin waveguiding layer. This is clearly seen in Fig. 3(a), showing the frequency response of the system. At frequencies well above that of the lower frequency pole $\left(2 \pi C_{\mathrm{b}} \times R_{\mathrm{s}}\right)^{-1} \approx 18 \mathrm{~Hz}$, the capacitor $C_{\mathrm{b}}$ can be effectively substituted by a dc voltage generator, which, in fact, makes the temperature in the thermal nucleus of the bulk insensitive to the high-speed external excitations. Therefore, above this frequency the response of the system is dominated by the pole associated to the superficial waveguide volume, and approximated by

$$
\begin{aligned}
H(s) & =\frac{V_{\text {out }}(s)}{I(s)}=\frac{H(0)}{1+s \cdot \tau_{\mathrm{w}}} \\
& =\frac{R_{\mathrm{c}} R_{\mathrm{b}} /\left(R_{\mathrm{c}}+R_{\mathrm{b}}+R_{\mathrm{w}}\right)}{1+s C_{\mathrm{w}} R_{\mathrm{b}}\left(R_{\mathrm{c}}+R_{\mathrm{w}}\right) / R_{\mathrm{c}}+R_{\mathrm{b}}+R_{\mathrm{w}}}\left[\frac{V}{A}\right]
\end{aligned}
$$

with $\tau_{\mathrm{w}} \approx 1.02 \times 10^{-6} \mathrm{~s}$, which corresponds to a cutoff frequency $\left(2 \pi \tau_{\mathrm{w}}\right)^{-1}$ of $155 \mathrm{kHz}$. Clearly, the application of a current pulse to the EEN (i.e., of a heat pulse, at a fixed power, to the real thermal system) at higher frequencies would, in fact, produce a significantly distorted thermal, and therefore optical, output signal. It should be also noted that in Eq. (7), $R_{\mathrm{b}}$ and $C_{\mathrm{w}}$ have a dominant role in determining the system bandwidth.

In addition, we observe that $\tau_{\mathrm{w}}$ does not depend on the modulator length because all $C$ 's are proportional to $L$,

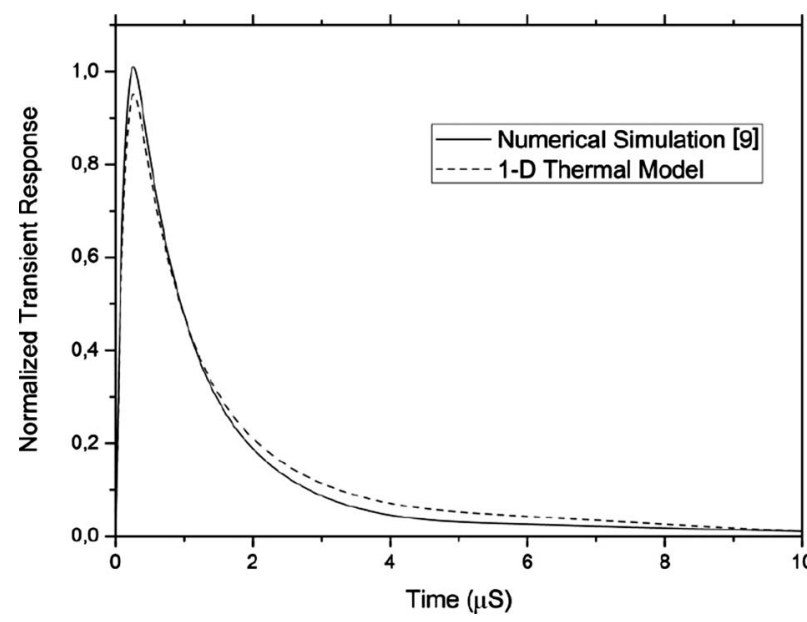

Fig. 4 Warm-up and cooldown transients as estimated from the simple thermal model of Fig. 2. The forcing signal is a 150-ns-wide, 2 A-peak, current pulse, which thermally corresponds to a $2 \mathrm{~W}$-peak thermal drive. The transient response calculated by numerical simulations ${ }^{6}$ is also shown for comparison.

while all $R$ 's are proportional to $L^{-1}$. The normalized network response to a 150 -ns-wide, 2-A peak, current pulse (corresponding to a $2-\mathrm{W}$ peak, thermal drive) is shown for example in Fig. 4. As noted above, this response is not dependent on the device length. In the same plot, the transient response to the same current pulse, calculated by means of two-dimensional thermal numerical simulations 6 is also shown, demonstrating that the simplified analytical model provides reliable results. As can be seen, the cooldown transient can be considered in practice extinguished within a few $\tau_{\mathrm{w}}$ 's. In the case of a 300- $\mu$ m-long cavity, the maximum voltage at the output node would be $6.7 \mathrm{~V}$, corresponding to a temperature deviation at the waveguide center of $6.7^{\circ} \mathrm{C}$. A possible approach for improving the system response consists of modifying the network by adding a preprocessing section of the input pulse, as shown in Fig. 5. This section is, in fact, a derivator of the input signal $V_{\text {in }}$ (i.e., a high-pass filter).

It should be noted that, for our convenience, the input signal source is a voltage pulse generator. The new block has the role of adding a zero at the frequency of the dominant pole of the starting system, and a pole at a suitable higher frequency $\left(2 \pi \tau_{\mathrm{h}}\right)^{-1}$. This is clear from Fig. 3(b),

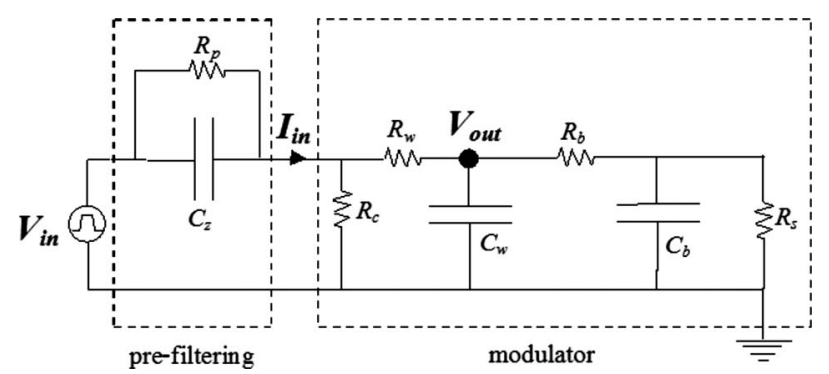

Fig. 5 Electrically-equivalent thermal model of the modulator including a prefiltering section. This section adds a zero at the frequency of the dominant pole of the base system and a pole at a suitable higher frequency, thus widening the overall network bandwidth. 

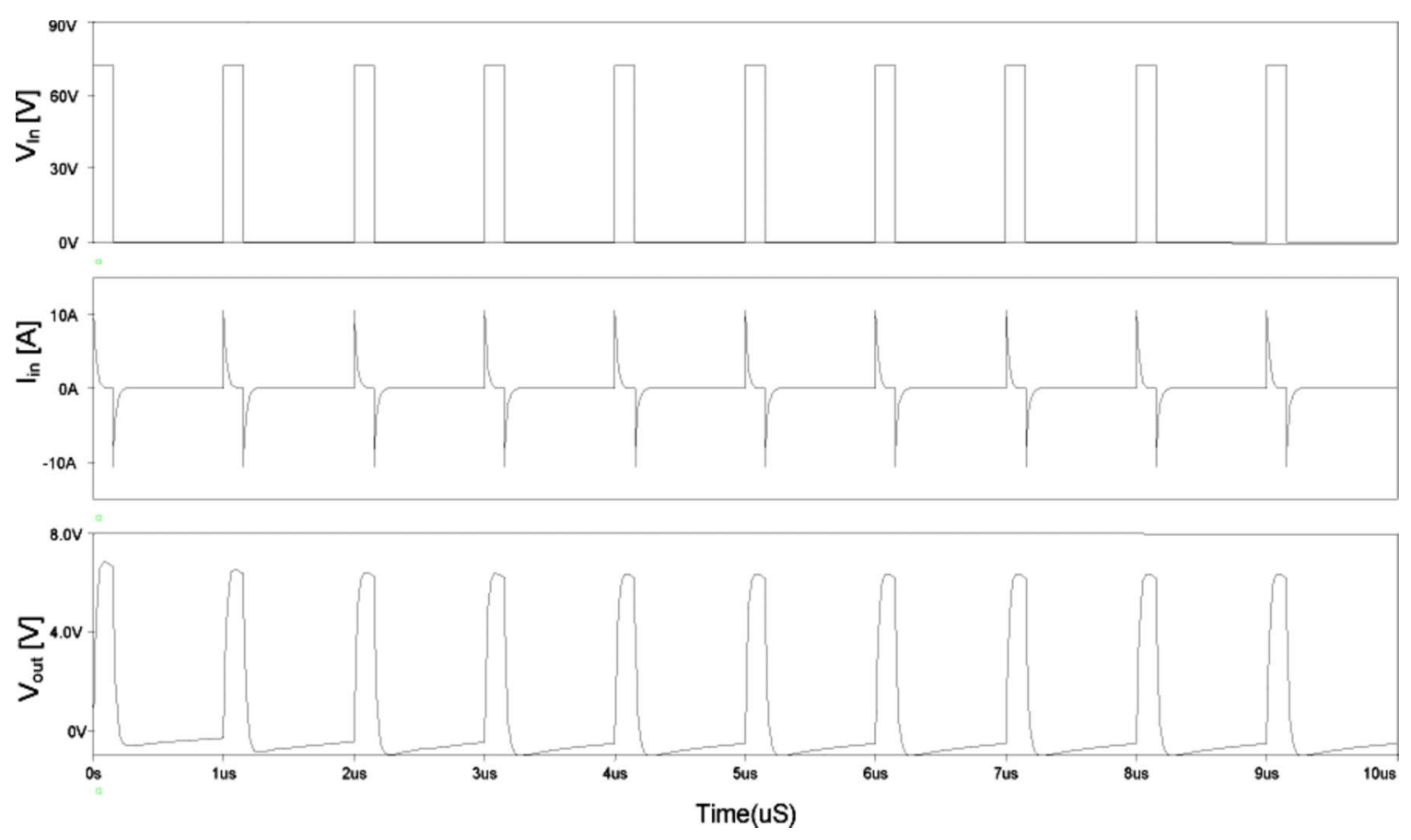

Fig. 6 Time evolution of $V_{\text {in }}, l_{\text {in }}$, and $V_{\text {out }}$ as estimated from the modified equivalent model, where a preprocessing section has been introduced. It should be noted that the top trace $\left(V_{\text {in }}\right)$ represents the forcing signal, the middle trace $\left(I_{\text {in }}\right)$ is equivalent to the transferred thermal power in (watts), while the bottom trace $\left(V_{\text {out }}\right)$ is the equivalent of the consequent temperature variation in the waveguide (in degree celsius).

where the spectral response of the system is shown after adding the prefiltering section, with $R_{\mathrm{p}}=300 \mathrm{~K} / \mathrm{W}$ and $C_{\mathrm{z}}$ $=3.4 \times 10^{-9} \mathrm{~J} / \mathrm{K}$. The $-3-\mathrm{dB}$ bandwidth increases from $155 \mathrm{kHz}$ to $\sim 5 \mathrm{MHz}$. The modified network response to a 150-ns-wide, 70-V-high, 1- $\mu$ s-period pulse sequence is shown in Fig. 6. Compared to Fig. 4, this time the shape of the output signal $V_{\text {out }}$ (i.e., of the temperature evolution at the waveguide center) well approximates a sequence of pulses.

On the same plot, the current provided to the original network section $I_{\text {in }}=\left(I_{R_{\mathrm{c}}}+I_{R_{\mathrm{w}}}\right)$ is reported (middle plot). As expected, it is in fact the derivative of the input signal. The positive pulse of this current signal delivers a given electrical charge (i.e., a thermal energy) to the system to bootstrap its output to the desired final amplitude (i.e., heats the waveguide core up to the desired temperature), while the negative pulse subtracts the same quantity of charge (i.e., of heat) to rapidly bring the system back to its original state. This approach does not have a thermal equivalent in practice. In fact, in no simple way can we apply negative heat pulses (i.e., cooling pulses) to the modulator. For this reason, we will hereafter propose to obtain the required heat flux $\left(I_{\text {in }}\right)$ by means of another approach.

Instead of introducing a refrigerating element on top, a viable solution can, in fact, consist of holding the modulator at a given higher average temperature with respect to the substrate temperature, thus enhancing the heat transfer between them. This can be obtained by supplying a continuous heat to the modulator. The modulation action will therefore consist of applying a given overheating to produce the necessary temperature increase, and then com- pletely switching off the heating to allow a more rapid cooling. A perfect balance between the supplied and extracted heat should be respected to allow the system rapidly recover its initial thermal state and to reject the drift of the modulator temperature. The validity of this approach was already empirically demonstrated by numerical simulations in Ref. 6, while a practical realization of the technique was shown on a Mach-Zehender device. ${ }^{16}$ It should be noted, in addition, that there is only an apparent system complication. On the contrary, with the proposed approach we eliminate, in principle, the need of expensive and inefficient Peltier heat pumps, always present in thermally controlled devices to hold the device at a fixed temperature.

\section{Simulation Results}

The device response to a heat pulse generated by electrical power dissipation in the top resistive film to induce the thermo-optical modulation depends on the initial temperature, the room temperature, and the substrate bottom temperature as described in previous sections. We define a rise time $t_{\text {on }}$ and a fall time $t_{\text {off }}$ to better describe the dynamic characteristics of the modulator: $t_{\mathrm{on}}$ represents the time it takes to drive the device from 10 to $90 \%$ of the desired final temperature in the heating phase. $t_{\text {off }}$ is the time interval necessary to allow the device to evolve between 90 and $10 \%$ of the high temperature in the cooling phase. If a series of heating pulses is applied with a period $T_{\mathrm{O}}$ shorter than $t_{\text {on }}+t_{\text {off }}$, then the device accumulates a residual quantity of heat per cycle, which is translated into a device average temperature drifts upward and in an unpredictable output, because of the mismatching of the refractive index. 


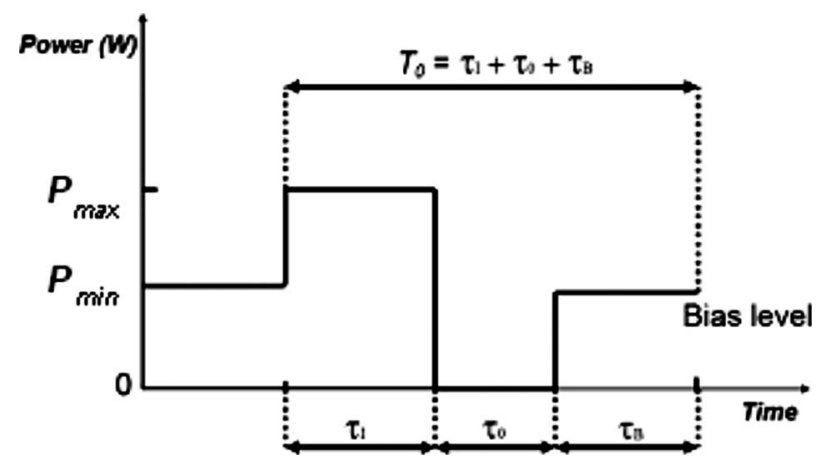

Fig. 7 Proposed modulation signal. When the bias signal is applied, the modulator is held at a stable temperature above room temperature.

To avoid this phenomenon, simulations indicate that for our device the period should last about $16 \mu \mathrm{s},{ }^{6}$ which implies a bit rate as low as $60 \mathrm{kbits} / \mathrm{s}$.

As demonstrated in Sec. 3, fastest transients can be obtained by applying a complex thermal driving signal consisting of a positive and negative heat spike. As a practical alternative, due to the impossibility of providing a functional cooling pulse, a continuous heat can be applied to the modulator at rest, by flowing a given current through its top heating film, which maintains the device at a given higher temperature, with the modulation action consisting of the application of an overcurrent, to produce the desired overheating, and a subsequent complete current switch-off to allow the fastest possible cooling. The simplest signal performing these characteristics is the one shown in Fig. 7.

It consists of $(i)$ a $P_{\min }$ bias level (middle level), realizing a thermal bias, which in steady-state conditions holds the active volume of the modulator at a higher average temperature with respect to the substrate bottom (for example, $125^{\circ} \mathrm{C}$ ); (ii) a $P_{\max }$ modulation pulse (high level), with width $\tau_{\text {I }}$ (iii) a power-off phase (low level) in which no power for a time $\tau_{\mathrm{o}}$ is applied to the polysilicon film in order to permit the heat flows between $131.7^{\circ} \mathrm{C}$ (i.e., $125+6.7^{\circ} \mathrm{C}$ ) and $25^{\circ} \mathrm{C}$ (substrate bottom temperature). Finally, the thermal bias is applied again at least for a time $\tau_{\mathrm{B}}$ before a new pulse can be applied. The signal period is therefore $T_{\mathrm{o}}=\tau_{\mathrm{I}}+\tau_{\mathrm{o}}+\tau_{\mathrm{B}}$.

If we assume $\tau_{\mathrm{I}}$ to be $150 \mathrm{~ns}$ (as for the case of Fig. 4), then $\tau_{\mathrm{o}}$ and $\tau_{\mathrm{B}}$ must be tuned for optimal device operation. Therefore, a set of simulations has been carried out to evaluate the minimum time parameters that ensure the complete recovery. We get $\tau_{\mathrm{O}}=105 \mathrm{~ns}$ and $\tau_{\mathrm{B}}=800 \mathrm{~ns}$, corresponding to $T_{\mathrm{o}}=1055 \mathrm{~ns}$. Because, in general, a modulation sequence can contain an unpredictable number of consecutive pulses, shorter periods are, in principle, forbidden if a thermal drift over a long observation time must be avoided in the structure. This rule is however not as stringent as it may seem.

This thermal transient response has been used to calculate the associated optical response by using Eq. (1). In practice, at each time step we calculate the actual refractive index $n_{\mathrm{Si}}$ as

$n_{\mathrm{Si}}=n_{\mathrm{Si}, \mathrm{o}}+\Delta n_{\mathrm{Si}}$,

where $n_{\mathrm{Si}, \mathrm{o}}$ is the refractive index at the bias level and

$\Delta n_{\mathrm{Si}}=1.86 \times 10^{-4} \Delta T$.

The modulator optical output is shown in Fig. 8(a).

\section{Circuit Design}

In order to test the proposed complex signal, a modulator driver IC that occupies an area of $4 \mathrm{~mm}^{2}$ in $0.8 \mu \mathrm{m}$ CMOS has been designed and fabricated. The block diagram is described in Fig. 9.

The target was to design an IC capable of converting an arbitrary sequence of $0-1$, at a given bit rate, into the ap-

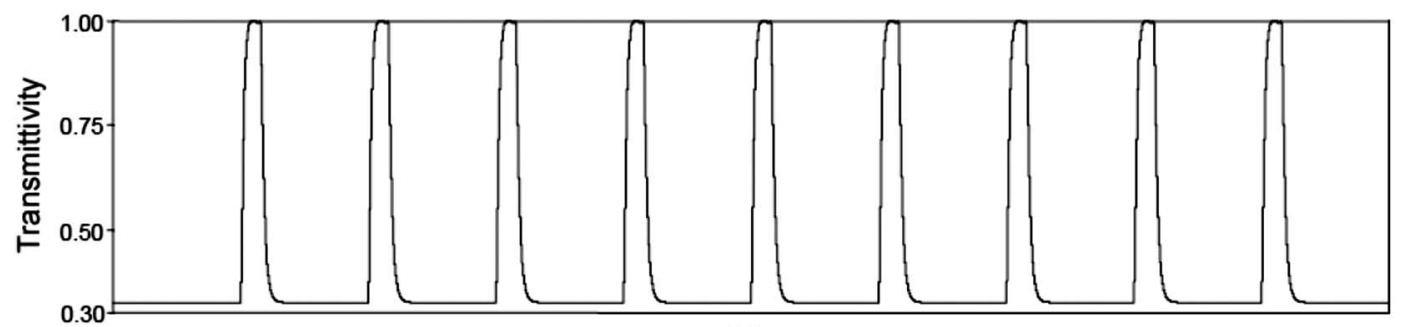

(a)

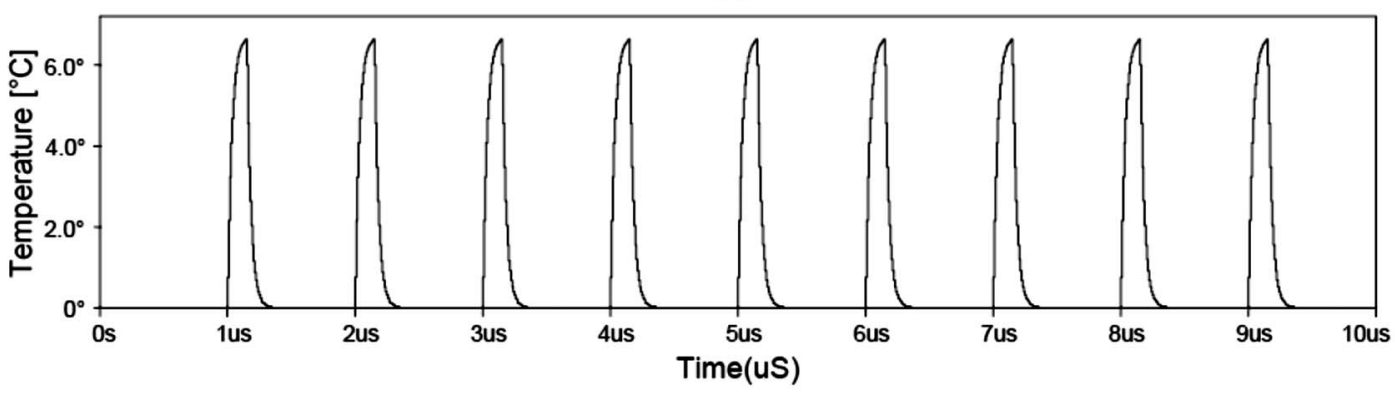

(h)

Fig. 8 Optical (a) and thermal (b) response of the modulator to a pulse sequence at a bit rate of $1 \mathrm{Mbits} / \mathrm{s}$. 

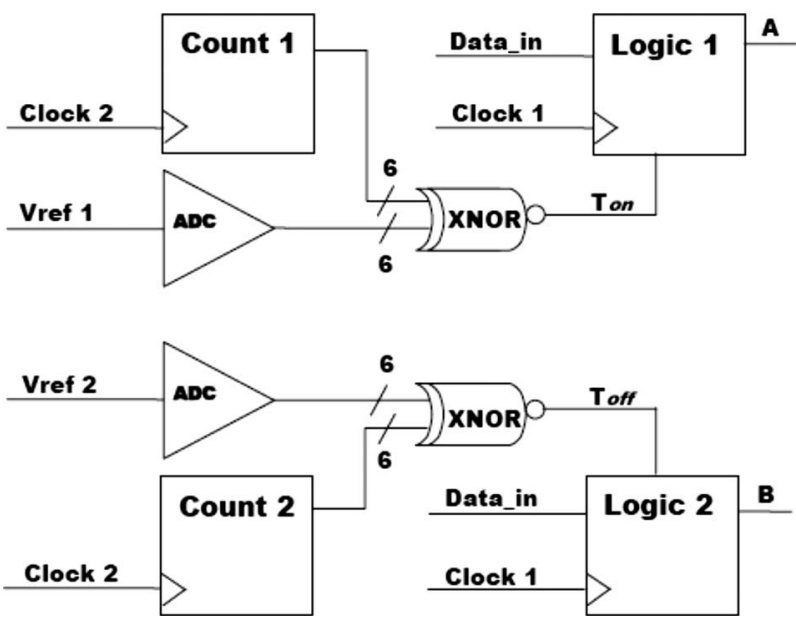

Fig. 9 Block diagram of the modulator driver IC.

propriate sequence of $0-1$ for the signals $\mathrm{A}$ and $\mathrm{B}$ in order to get the modulation strategy described above. The circuit has to work at various bitrates (user selectable), with an as-low-as-possible number of external components, and has to allow a fine adjustment of the previously defined time durations. The IC therefore provides the two logical signals, A and B, as outputs, which control the following power stage and the driving signal applied to the modulator pins.

The A output provides the driver signal with the $P_{\min }$ bias level (middle level), realizing the thermal bias. In practice, it sets the time period during which the current is applied to the modulator to obtain a higher average temperature with respect to the substrate bottom. The B output provides the driver signal with the modulation pulse (high level), $P_{\max }$. In practice, it sets the time period during which the overcurrent is applied to the polysilicon film in order to permit the overheating of the modulator volume.

LOGIC 1 and LOGIC 2 blocks take into account the input value DATA_IN and the heating and cooling phase time flag $\left(T_{\text {on }}\right.$ and $\left.T_{\text {off }}\right)$ to synchronize $\mathrm{A}$ and $\mathrm{B}$ output as required. The duration of $T_{\text {on }}$ and $T_{\text {off }}$ can be adjusted by external trimmers to allow the fine regulation and the optimum phase duration in the test phase. The two different clock signals permit another degree of freedom in the time setting. The die photograph is shown in Fig. 10.

\section{Power Stage}

The modulator driver supplies the CMOS signals A and B as described in Sec. 5. In order to match the time resolution required by $\tau_{\mathrm{I}}, \tau_{\mathrm{o}}$, and $\tau_{\mathrm{B}}$, a power stage has been designed and realized to translate the logic levels into the three power levels described in Sec. 4.

The adopted configuration consists of the direct connection of the outputs of the driver IC (A and B) to the gate of two MOS drivers. In defining the layout, a low-noise analog ground plane was realized and other precautions were taken in order to reduce inductive and capacitive parasitic effects. The two IXIS-RF Power MOS utilized, DE150201, are specifically designed for high-speed power switching, and exactly match the purpose of this test board. The connections of the driver's output to the differential con-

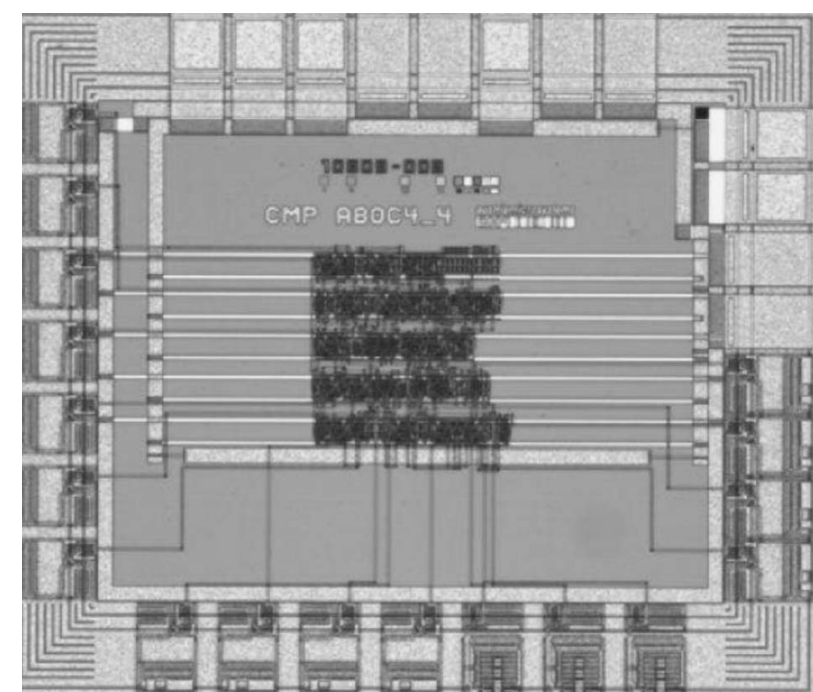

Fig. 10 Micrograph of the integrated driving circuit. Dimensions are $2 \times 2 \mathrm{~mm}^{2}$ approximately.

figuration of the two MOSFETS are shown in Fig. 11. The power supplied to the modulator in the different working phases are summarized in Table 4.

\section{Test}

The proposed method and fabricated IC were tested with an all-silicon waveguide integrated thermo-optic Fabry-Perot modulator. The device was already described in Ref. 6. It is

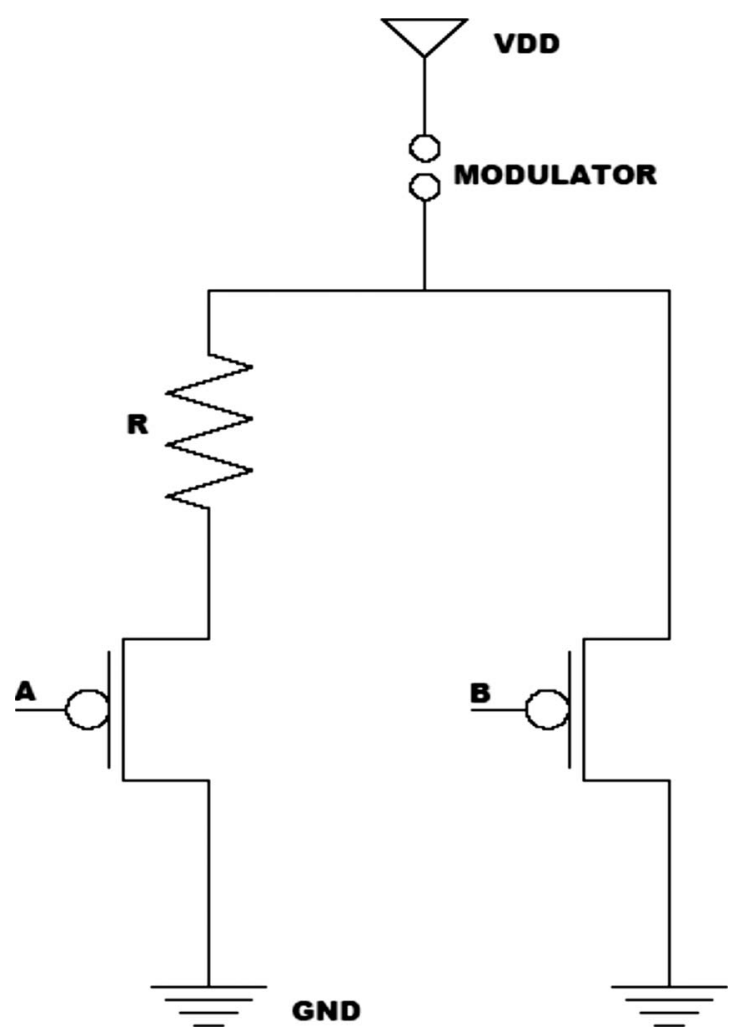

Fig. 11 Schematic of the power stage. The heating film on the top of the modulator is the actual load (MODULATOR). 
Table 4 Specification of the power applied to the modulator in the three possible configurations of the driving circuit outputs.

\begin{tabular}{ccc}
\hline \hline $\mathrm{A}$ & $\mathrm{B}$ & Applied Power \\
\hline 0 & 0 & 0 \\
1 & 0 & $P_{\min }=400 \mathrm{~mW}$ \\
0 & 1 & $P_{\max }=600 \mathrm{~mW}$ \\
\hline \hline
\end{tabular}

embedded in a 8 - $\mu \mathrm{m}$-wide, 8 - $\mu \mathrm{m}$-thick, rib waveguide, with a rib height of $2.2 \mu \mathrm{m}$. In spite of its large cross section, the waveguide is single mode (TE). It should be noted that the device is an all-silicon one [i.e., the light confinement at bottom is sustained by the small refractive index change between an undoped silicon layer (core) and a heavily doped silicon layer (bottom cladding)]. This helps removing the thermal bottleneck typical of SOI-based thermo-optical devices. ${ }^{2}$ The modulator length $(250 \mu \mathrm{m})$ is defined by means of deep-trench etching. The resistive film on top is a 300 -nm-thick polysilicon layer, showing a resistance of $1.2 \mathrm{k}$. Figure 12 reports a SEM image of the integrated modulator.

A laser source operating at $1550 \mathrm{~nm}$ was injected through a single-mode fiber into the all-silicon waveguide. The modulated optical signal was collected at the opposite side of the device by an infrared (IR) photodiode and then analyzed using a digital oscilloscope. In Fig. 13, the modulation patterns obtained with a standard modulating pulse [Fig. 13(a)] and the three-level signal [Fig. 13(b)] are compared. The two plots are normalized; however, the modulation depths are the same. In the first case, assuming that the modulator is in the off state (on state) when the optical output is $<10 \%(>90 \%)$ of its dynamic, we get that the transition times $t_{\mathrm{off}}$ and $t_{\mathrm{on}}$ are measured to be 17 and $9 \mu \mathrm{s}$, respectively. It should be noted, however, that at the considered driving frequency, the optical output evolution is not complete yet, both for the rising and in the falling phases, and therefore, an increase of the driving frequency would imply a lower modulation depth.

In the case of the three-level signal [Fig. 13(b)], we assume that the "low" optical output value is that transmitted during the application of the bias level as defined in Fig. 7 , or rest period. Because of an overchilling occurring in the zero power phase $(A=0, B=0)$, Fig. 13(b) indeed reveals that the signal actually goes below this level for a short time interval, a phenomenon that should however not have an impact on the transmission and that can be reduced through a finer tuning of the average device temperature. With this modulation scheme, and for $\tau_{\mathrm{I}}=20 \mu \mathrm{s}, \tau_{\mathrm{o}}$ $=10 \mu \mathrm{s}$, and $\tau_{\mathrm{B}}=20 \mu \mathrm{s}$, we now get $t_{\mathrm{off}}=5 \mu \mathrm{s}$ and $t_{\mathrm{on}}$ $=8 \mu \mathrm{s}$. It should be noted that, in this case, the optical output evolution is complete both in the rising and falling phases, and therefore, an increase of the driving frequency would not imply a modulation depth reduction. In addition, the $t_{\text {off }}$ of the optical output appears to be limited by the $t_{\text {off }}$ of the driving signal [top trace of Fig. 13(b)], limited in turn by the output resistance of the output power stage.

The energy per cycle provided to the modulator decreases linearly with frequency because, for decreasing fre-

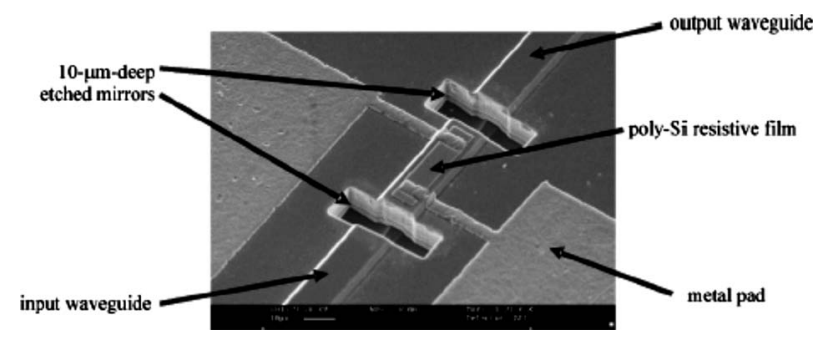

Fig. 12 Scanning electron micrograph of the actual thermo-optical modulator.

quencies, the $\tau_{\mathrm{I}}, \tau_{\mathrm{o}}$, and $\tau_{\mathrm{B}}$ times are reversely scaled accordingly. This implies that the average power provided to the modulator is constant $\left(P_{\mathrm{av}} \approx 400 \mathrm{~mW}\right)$. It should be considered that most of this power is spent to hold the device at a higher average temperature, not to get modulation. This power is not very different from that required for the otherwise necessary thermal regulation of the device obtained by means of inefficient Peltier heat pumps.

\section{Conclusion}

The problem of the heat transients in high-speed thermally controlled optical devices has been described by means of a simple analytical model based on the electrical equivalent network of thermal structures. The model has allowed showing that the bandwidth of a silicon-based, waveguideembedded, thermo-optic micromodulator can be assumed as limited by the thermal capacitance of the waveguiding layer, which slows down the free evolution of the device cooling phase. With the support of this mixed thermal and optical analysis, tuned to a real modulator, we have determined the time-intensity characteristics of a three-level driving signal that can significantly cut the switch-off time. The modulator driver has been fabricated as a custom $0.8-\mu \mathrm{m}$ CMOS IC and tested on a $250-\mu \mathrm{m}$-long FabryPerot silicon micromodulator, showing a reduction of the cooling phase duration from 17 to $5 \mu \mathrm{s}$. The proposed model and approach could be applied to several other simi-

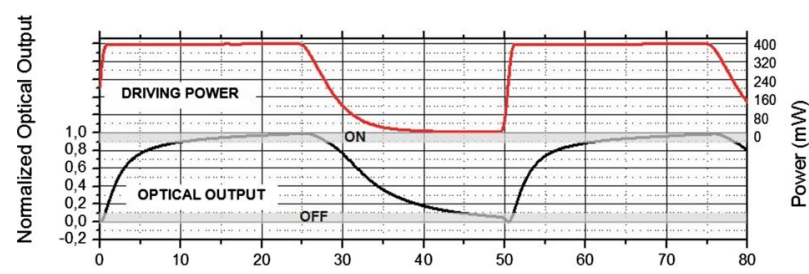

(a)

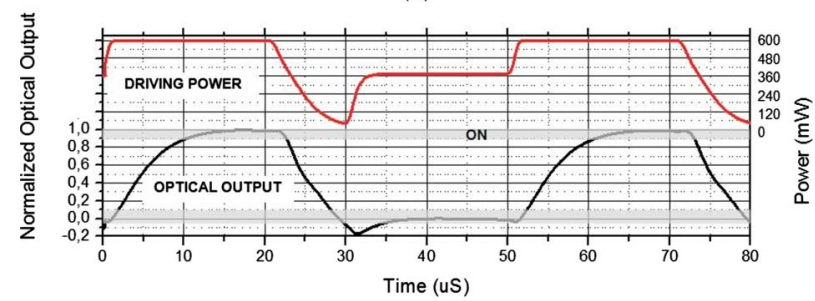

(b)

Fig. 13 (a) Modulator optical output when a two level modulation is applied; (b) Modulator optical output when the modulation sequence of Fig. 8 is applied. 
lar problems occurring in integrated optics, where fast temperature variations are required (e.g., in tunable lasers) or even when they are the effect of stray phenomena.

\section{References}

1. R. A. Soref, "Silicon-based optoelectronics," in Proc. IEEE Vol. 81(12), 1687-1706 (Dec. 1993).

2. G. V. Treyz, "Silicon Mach-Zehnder waveguide interferometers operating at $1.3 \mu \mathrm{m}, "$ Electron. Lett. 27(2), 118-120 (Jan. 1991).

3. R. A. Mayer, K. H. Jung, W. D. Lee, D.-L. Kwong, and J. C. Campbell, "Thin-film thermo-optic GexSi1-x Mach-Zehnder interferometer," Opt. Lett. 17(24), 1812-1814 (Dec. 1992).

4. U. Fischer, T. Zinke, B. Shuppert, and K. Petermann, "Singlemode optical switches based on SOI waveguides with large cross-section," Electron. Lett. 30(5), 406-408 (Mar. 1994).

5. G. Cocorullo, M. Iodice, I. Rendina, and P. M. Sarro, "All-silicon thermo-optic micro-modulator," in Proc. 25th European Solid State Device Res. Conf., H. C. de Graff and H. van Kranenburg, Eds., pp. 651-654, The Hague, Netherlands, Edition Frontiers, Gif sur Yvette, France (1995).

6. L. Gu, W. Jiang, X. Chen, and R. T. Chen, "Thermooptically tuned photonic crystal waveguide silicon-on-insulator Mach-Zehnder interferometers," IEEE Photonics Technol. Lett. 19(5), 342-344 (March 2007).

7. J. Yang, Q. Zhou, and R. T. Chen, "Polyimide-waveguide-based thermal optical switch using total-internal-reflection effect," Appl. Phys. Lett. 81(16), 2947-2949 (2002).

8. X. Wang, B. Howley, M. Y. Chen, and R. T. Chen " $4 \times 4$ nonblocking polymeric thermo-optic switch matrix using the total internal reflection effect," IEEE J. Sel. Top. Quantum Electron. 12(5), 9971000 (Sept./Oct. 2006)

9. M. Iodice, F. G. Della Corte, I. Rendina, P. M. Sarro, and M. Bellucci, "Transient analysis of a high-speed thermo-optic modulator integrated in an all-silicon waveguide," Opt. Eng. 42(1), 169-175 (Jan. 2003)

10. G. Cocorullo, M. Iodice, I. Rendina, and P. M. Sarro, "Silicon thermooptical micromodulator with 700-kHz-3-dB bandwith," IEEE Photonics Technol. Lett. 7, 363-365 (Apr. 1995).

11. G. Cocorullo, F. G. Della Corte, I. Rendina, and A. Cutolo, "New possibilities for efficient silicon integrated electro-optical modulators," Opt. Commun. 86(2), 228-235 (Nov. 1991).

12. The Institution of Electrical EngineersProperties of Silicon, pp. 3, 35, 37, INSPEC, New York (1988).

13. G. Cocorullo and I. Rendina, "Thermo-optical modulation at $1.5 \mu \mathrm{m}$ in silicon etalon," Electron. Lett. 28(1), 83-85 (Jan. 1992)

14. S. P. Pogossian, L. Vescan, and A. Vonsovici, "The single mode condition for semiconductor rib waveguides with large cross section," $J$. Lightwave Technol. 16(10), 1851-1853 (Oct. 1998)

15. G. N. Ellison, "Maximum thermal spreading resistance for rectangular sources and plates with nonunity aspect ratios," IEEE Trans. Compon. Packag. Technol. 26(2), 493-454 (June 2003).

16. M. Harjanne, M. Kapulainen, T. Aalto, and P. Heimala, "Sub- $\mu$ s switching time in silicon-on-insulator Mach-Zehnder thermooptic switch," IEEE Photonics Technol. Lett. 16(9), 2039-2041 (Sept. 2004).

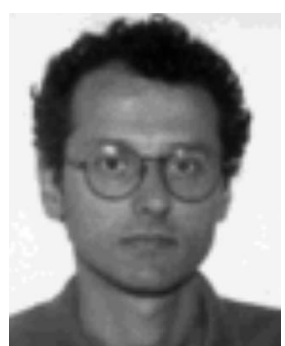

Francesco G. Della Corte received the Laurea in electronic engineering from the "Federico II" University of Napoli, Italy, in 1988. After a two-year fellowship at the Institute for Research on Electromagnetism and Electronic Components of the National Research Council, Italy, in 1990, he was a visiting researcher at the Institute for High Frequency Technology of the Technical University of Darmstadt, Germany. From 1991, he has been working on amorphous silicon devices at ENEA, Portici, Italy. In 1993, he joined as project leader the National Consortium for the Optoelectronic Technologies of InP (OPTEL) in Brindisi, Italy. From 1994 to 2001, he has been a researcher at the Institute for Research on Electromagnetism and Electronic Components of the National Research Council, Italy, where he has been responsible for the Electronic Components Division. From 2001, he is a professor of electronics at the "Mediterranea" University of Reggio Calabria, Italy. His main research interests are optoelectronic sensors and microsystems, bipolar device modeling, heterojunction devices, thin film silicon devices, and the characterization of optoelectronic materials.

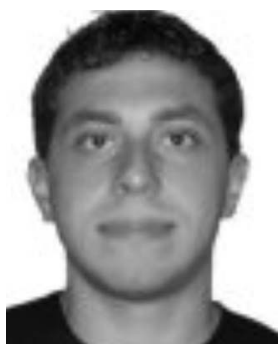

Massimo Merenda received his BS, MS, and $\mathrm{PhD}$ in electronic engineering from the "Mediterranea" University of Reggio Calabria, Italy, in 2002, 2005, and 2009, respectively. From 2003 to 2005, he was a fellow at Institute for Microelectronics and Microsystems of the National Council of Research (CNR), Naples, Italy. His main research interests are application specific IC design, embedded system design, silicon sensor, and design of VLSI systems for radio-frequency identification devices.

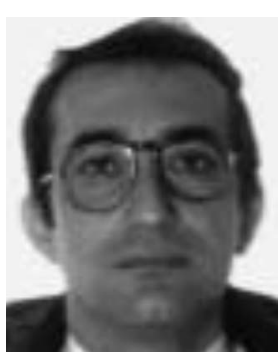

Giuseppe Cocorullo received his Dr. Eng. degree in electronics from the University of Naples, Naples, Italy, in 1978. From 1983 to 1992, he was with the National Council of Research, IRECE Institute, Naples, Italy, where he was in charge of the Microelectronic Department. He has been an associate professor of electronics at the University of Calabria, Rende, Italy, since 1992. In 2000 , he was appointed a full professor of electronics at the University of Calabria. His main research interests include the fields of silicon optoelectronics and application specific IC design.

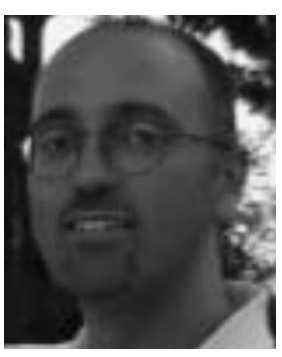

Mario lodice received his $\mathrm{MSc}$ and $\mathrm{PhD}$ in electronic engineering at the University " $\mathrm{Fe}$ derico II," Napoli, Italy, in 1991 and 1997, respectively. From 1992 to 1994 , he was on scholarship to the IRECE National Research Council (CNR), Napoli, where he was involved in integrated optics and optoelectronics and on laser characterization of electrooptic materials. From 1993 to 1994 , he was a visiting researcher at the Delft Institute of Microelectronics and Submicron Technologies, Technical University of Delft, The Netherlands, where he worked on microelectronics technologies and on thermal sensors design. From 1995 to 1997, he was with the Department of Electronic Engineering, University of Napoli "Federico II," working on modeling and design of silicon electro-optic modulator. During the second half of 1996, he visited the Department of Electrical Engineering, Princeton University, Princeton, NJ, where he worked on the thermal analysis of flat panel displays. Currently, he is a senior researcher at Institute for Microelectronics and Microsystems (IMM)CNR, where he works on integrated optical circuit and devices, and optical sensors. He also taught electronics at the University of Reggio Calabria, from 1997 to 2001, and sensors at the University of Calabria, Cosenza, Italy, in 2002.

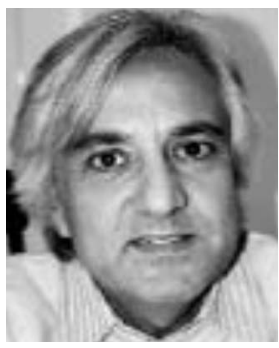

Ivo Rendina is a research director with the National Council of Research (CNR), Naples, Italy, where he has been the director of the Institute for Electromagnetism and Electronic Components and is currently responsible for the Department of Napoli, Institute for Microelectronics and Microsystems, Naples, Italy. $\mathrm{He}$ also teaches electronics and optoelectronics at the University of Calabria, Cosenza, Italy. His present research interests are in the field of silicon optoelectronics and microsystems. He is a member of the "Consiglio di Presidenza" of the Società Italiana di Ottica e Fotonica and SPIE. 
Della Corte et al.: Modulation speed improvement in a Fabry-Perot thermo-optical modulator...

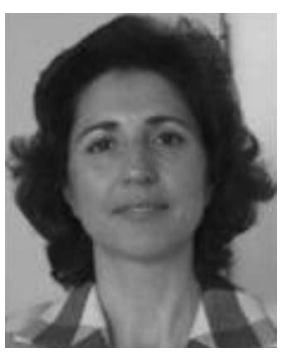

Pasqualina M. Sarro received the Laurea degree (cum laude) in solid-state physics from the University of Naples, Naples, Italy, in 1980 , and the PhD degree in electrical engineering from Delft University of Technology, Delft, The Netherlands, in 1987 , where her thesis dealt with infrared sensors based on integrated silicon thermopiles. From 1981 to 1983 , she was a postdoctoral fellow with the Photovoltaic Research Group, Division of Engineering, Brown University, Providence, Rhode Island. She then joined the Delft Institute

of Microsystems and Nanoelectronics, Delft University of Technology, where she is responsible for research on integrated silicon sensors and microelectromechanical systems (MEMS) technology. In December 2001, she became the A. van Leeuwenhoek Professor and, since 2004, has been the head of the Electronic Components, Materials and Technology Laboratory. She has authored or coauthored more than 350 journal and conference papers. 